\title{
Open Trial with Clofazimine in the Management of Recurrent Lepra Reaction and of Sulphone Sensitive Cases: A Preliminary Report*
}

\author{
K. RAMANUJAM, C. G. S. IYER AND G. RAMU \\ Central Leprosy Institute, P.O. Tirumani, Chingleput 603001, S. India
}

\begin{abstract}
An open trial to assess the value of clofazimine in the management of cases of lepromatous leprosy with recurrent lepra reaction and those who are sulphone sensitive was commenced in February, 1973. Thalidomide was used as the drug in the control subjects. Up to the end of August, 1974, 61 cases have been admitted into the study. In the majority of instances the drug had been found to be effective. In 3 instances clofazimine failed to confer beneficial effects. Six cases in whom the maintenance dose of the drug was stopped after 52 weeks of continued control of the reactive state, have not revealed any recurrence of reaction.
\end{abstract}

\section{The Investigation}

A study was undertaken to assess the therapeutic value of clofazimine (CLF) with the following objectives, using Thalidomide (TLD) as the control drug: (a) To assess the value of clofazimine in the management of recurrent lepra reaction and (b) to ascertain whether this drug can increase tolerance to DDS in cases of lepromatous leprosy, who are sulphone sensitive and prone to recurrent reaction.

\section{Plan of the study}

The investigation has been planned as a continuous one in order to deal with both the objectives (a) and (b). Cases of lepromatous leprosy subject to recurrent episodes of lepra reaction will receive clofazimine or thalidomide during Part (a) of the investigation, lasting for 8 weeks. It is expected that the reactive episodes will be controlled within the 8 weeks' period, with either of these 2 drugs. Thereafter the same patient serves as subject for Part (b) of the trial. If any of

* This investigation received financial support from the WHO. 
these cases fail to respond to therapy in the 8 weeks' period, the drug is considered a failure, and the patient taken off the trial. Once the patients are transferred to Part (b) of the trial and the reaction is under control, steroid dependent patients are weaned from steroid, DDS treatment is inducted and gradually increased and the dose of clofazimine/thalidomide reduced progressively until the case on clofazimine continues to receive a maintenance dose of $100 \mathrm{mg}$ of the drug per day and those on thalidomide, a dose of 25 to $50 \mathrm{mg} / \mathrm{day}$. The dose of DDS is increased to the maximum dose of $10 \mathrm{mg} / \mathrm{kg}$ body weight/week or to the limit of tolerance.

\section{Subjects for the study}

Adult male patients with lepromatous leprosy who had become subjects of recurrent reactive episodes and were sulphone sensitive constituted the subjects for the study. Patients who had become steroid dependent for the control of their reactive states were also eligible for the trial. Allocation of subjects to the trial (clofazimine) or control (thalidomide) group was done by randomization.

\section{Dosage}

In Part (a) of the investigation clofazimine was administered in a dose of $100 \mathrm{mg} 3$ times a day for the maximum period of 8 weeks. The patients in the control group received $100 \mathrm{mg}$ of thalidomide thrice a day for the same period.

In Part (b) of the trial induction of specific treatment with DDS was commenced with a small dose and gradually built up as indicated above and the dose of anti-reaction drug, clofazimine or thalidomide, was progressively reduced till the patients received a maintenance dose of $100 \mathrm{mg}$ of clofazimine or 25 to $50 \mathrm{mg}$ of thalidomide per day.

\section{Duration of trial}

The duration of Part (a) of the trial was for 8 weeks and of Part (b), 52 weeks.

\section{Side-effects}

Occurrence of side-effects in respect of the drugs was recorded in suitably drawn up proformae.

\section{Records}

Details in respect of the frequency, duration and severity of reactive episodes prior to entry into the study, details of reactive phases while in the trial, clinical and bacteriological progress under treatment and finding of laboratory investigation such as haemogram, urinalysis and liver function tests were recorded in suitable proformae.

\section{Laboratory investigations}

The preliminary investigation consisted of haemogram, urinalysis, stools examination, biochemical investigations, BI and MI, and skin biopsy. All the investigations except the last were repeated once a quarter. Skin biopsy was repeated as and when necessary. 
Assessment of progress

The progress of cases under treatment was suitably recorded and the results assessed according to pre-determined criteria. For Part (a) of the investigation, the progress of cases in relation to the control of frequency and severity of reactive episodes was recorded. For Part (b), the periodical assessment included the presence or absence of reactive episodes, ability to tolerate DDS with reference to its dosage and continuity of treatment, and clinical and bacteriological progress under such treatment.

\section{Removal from the study}

Cases who failed to register a satisfactory response within 8 weeks in Part (a) of the investigation and those unable to tolerate even minimum doses of DDS in Part (b) of the study were taken out of the study and counted as failures.

\section{Findings of the study}

The investigation was commenced on 20 February, 1973. Up to the end of August, 1974, 61 cases were included in the study. Of these 61 cases, 52 who had been on the trial for more than 8 weeks have been taken up for analysis. The categorization of these 52 cases with regard to the chief manifestation of reactive episode is as under:

Cases of recurrent Lepra Reaction (RLR) with mostly skin manifestations: Cases of recurrent Lepra Reaction (RLR) with mostly skin manifestations

and steroid dependent:

Cases of Recurrent Pustular Lepra Reaction:

Cases of Recurrent Pustular Lepra Reaction and steroid dependent:

Cases of RLR with neuritis as the main manifestation:

Cases of RLR with neuritis as the main manifestation and steroid dependent:

Case of RLR with predominantly osseous manifestation and steroid dependent:

Case of RLR with predominantly lymph adenitis:

Of the 61 cases included so far, 5 cases failed to respond to treatment during 8 weeks of Part (a) of the trial-3 on clofazimine and 2 on thalidomide, and hence were taken out of the study. Five cases ( 2 on clofazimine and 3 on thalidomide) absconded after recording very satisfactory progress.

Six cases receiving clofazimine and 7 cases thalidomide in maintenance doses, and DDS in the optimum dose, were taken off the anti-reaction drug (clofazimine or thalidomide) after the reactions were controlled, and remained controlled for 52 weeks. The cases who were on clofazimine continue to be free from reaction. All the 7 cases who had been on thalidomide have shown a relapse of the reactive state.

The findings of the study up-to-date indicate:

(i) Both clofazimine and thalidomide possess anti-inflammatory properties and are generally capable of controlling recurrent reactive episodes in lepromatous leprosy, irrespective of the predominant manifestation of reaction, except for pustular skin lesions which called for the concurrent administration of antibiotics.

(ii) Of the 2 drugs, thalidomide appears to exert the desired ef fect quicker. 
(iii) Both the drugs permit weaning of patients from steroid dependence.

(iv) Both the drugs increase the tolerance of patients to DDS.

(v) In view of the fact that clofazimine takes as long as 8-12 weeks to exert its anti-inflammatory effect, in very severe reactions or where painful manifestations are predominant it was mandatory to supplement clofazimine initially with cortico-steroids to obtain quick relief.

(vi) Reappearance of manifestations of reaction were observed in an occasional case with both the drugs when the dose was being reduced, or while the patient was on maintenance dose of either drug.

(vii) While generally either drug was effective in controlling recurrent reactive episodes, there were instances where they failed to achieve the desired effect.

(viii) Bacteriologically, in the subjects who had been on the trial for 6 to 19 months and receiving DDS in conventional dose, improvement was observed in both the groups, this being more appreciable in the clofazimine group.

(ix) Side-effects included pigmentation of the skin and mucous membrane, colouration of urine, stools and sweat and development of ichthyotic skin over the extremities in cases on clofazimine; dryness of nasal and buccal mucosa, somnolence and very occasionally bradycardia with or without irregular pulse in cases receiving thalidomide. In none of the cases were the side-effects severe enough to warrant interference with the continued administration of the drug.

(x) The follow-up of the 13 cases in whom the anti-reaction drug was withdrawn after control of reactions showed that the beneficial effect conferred by clofazimine are more long-lasting, presumably due to retention of the drug in the RE cells, these acting as depots of clofazimine from which the drug was released slowly thereby exerting its reaction suppressing effect. On the other hand, the anti-reaction properties of thalidomide seem to be short lived as evidenced by the recurrence of reaction in all the 7 cases soon af ter the cessation of the drug. 